\title{
Correction to: Association of CXCR2 genotype variations with HCV clearance in a Chinese population
}

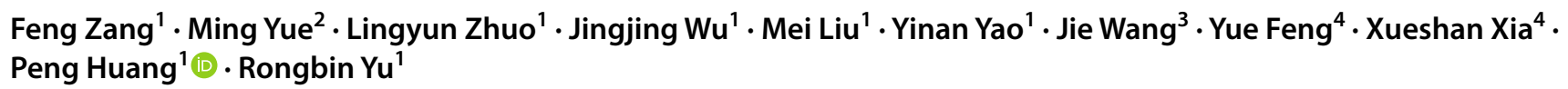

Published online: 8 December 2018

(c) Springer-Verlag GmbH Austria, part of Springer Nature 2018

Correction to: Archives of Virology (2018) 163:2711-2718
https://doi.org/10.1007/s00705-018-3872-0

Unfortunately, the funding statement was published with error in original publication and is corrected here.

Second paragraph of "Funding" should read as "Natural Science Foundation of Jiangsu Province (BK20171054, BK20151026)...”.

The original article can be found online at https://doi.org/10.1007/ s00705-018-3872-0.

Peng Huang

huangpeng@njmu.edu.cn

$\triangle$ Rongbin Yu

rongbinyu@njmu.edu.cn

1 Department of Epidemiology and Biostatistics, School of Public Health, Key Laboratory of Infectious Diseases, Nanjing Medical University, Nanjing 211166, China

2 Department of Infectious Diseases, The First Affiliated Hospital of Nanjing Medical University, Nanjing 210029, China

3 Department of Basic and Community Nursing, School of Nursing, Nanjing Medical University, Nanjing 211166, China

4 Faculty of Life Science and Technology, Kunming University of Science and Technology, Kunming 650500, China 